\title{
A young patient with multisystem complications after cytomegalovirus infection
}

\author{
Swaroopa Pulivarthi, Murali Krishna Gurram ${ }^{1}$ \\ Departments of Research, ${ }^{1}$ Internal Medicine, Health East Care System, Saint Paul, Minnesota, USA
}

\begin{abstract}
We are describing a case of an 18 -year-old male patient with cytomegalovirus (CMV) associated guillain-barre syndrome (GBS) who presented with an acute onset of generalized weakness and numbness in the extremities, dysphagia, and facial diplegia, followed by respiratory failure, which led to mechanical ventilation. He had positive immunoglobulin $\mathrm{G}$ and immunoglobulin $\mathrm{M}$ antibodies against $\mathrm{CMV}$, and $\mathrm{CMV}$ polymerase chain reaction was positive with <200o copies of deoxyribonucleic acid. Human immunodeficiency virus test was negative. He received a course of ganciclovir, intravenous immunoglobulin, and plasmapheresis. After improving from acute episode, patient was transferred to a rehabilitation facility for physical and occupational therapy. At the rehabilitation facility, he exhibited signs of acute abdomen with pain in the left upper quadrant secondary to peritonitis from dislodged gastrostomy tube and underwent exploratory laparotomy. During the hospital course he was found to have splenic infarct and colitis on the computed tomography of abdomen. This case showed an immunocompetent young patient with multisystem complications including guillain-barre syndrome (GBS), splenic infarct, hepatitis, and colitis due to CMV.
\end{abstract}

Key words: Cytomegalovirus, guillain-barre syndrome, hepatitis, splenic infarct

\section{Introduction}

Cytomegalovirus (CMV) associated guillain-barre syndrome (GBS) accounts up to $15 \%$ of all GBS cases..$^{[1]}$ The clinical manifestations of GBS associated with CMV occur often after respiratory tract symptoms, preferentially affects young women ( $<35$ years), and are frequently associated with sensory loss..$^{[2]}$ The clinical presentation of acute $\mathrm{CMV}$ infection include infectious mononucleosis syndrome (i.e., fever, rash, sore throat, atypical lymphocytes in the peripheral blood, monocytosis, thrombocytopenia, and elevated liver enzymes), pneumonitis, retinitis, colitis, and any other organ involvement. ${ }^{[3-5]} \mathrm{CMV}$ infection may rarely present with severe organ specific complications in immunocompetent hosts. The frequent presentation is severe hepatitis. ${ }^{[6,7]}$ The second frequent complications in immunocompetent patients are central nervous system disorders such as meningitis, encephalitis, myelitis, nerve

\begin{tabular}{|l|l|}
\hline \multicolumn{2}{|c|}{ Access this article online } \\
\hline Quick Response Code: & Website: \\
\hline & www.ruralneuropractice.com \\
\cline { 2 - 2 } & \\
\hline & \\
\hline
\end{tabular}

palsies, and GBS. ${ }^{[8]}$ The cases involving multiple systems due to CMV are very rare. We are describing a case of CMV-induced hepatitis, colitis, splenic infarct, and GBS in an 18-year-old male with intact immune status.

\section{Case Report}

An 18-year-old previously healthy male presented to the clinic with the complaints of sore throat and generalized malaise. The patient denied any history of alcohol or drug use. He denied any history of recurrent infections. The patient had no history of bleeding diathesis. His platelet count was normal. He had a negative rapid monospot and rapid strep antigen test. He recovered from acute illness in 3 days.

He noticed dysphagia and paresthesias of both lower extremities and weakness in all extremities (lower $>$ upper, proximal $>$ distal) one day after recovering from sore throat. On neurological examination, patient had bilateral facial nerve palsy and bulbar palsy. Motor strength was grade 1 in lower extremities and grade 2 in upper extremities. Muscle tone of the limbs was decreased. Deep tendon reflexes on the upper limbs were diminished and absent on the lower limbs. 
Blood chemistry was normal, except for total bilirubin of $2.3 \mathrm{mg} / \mathrm{dL}$, alanine transferase and aspartate transferase levels of $87 \mathrm{IU} / \mathrm{L}$ and $67 \mathrm{IU} / \mathrm{L}$ respectively, indicating hepatitis, which may be due to acute CMV infection. Test for human immunodeficiency virus was negative. His serology was positive for CMV immunoglobulin M $(\mathrm{IgM})$ and Immunoglobulin G (IgG) antibodies. CMV polymerase chain reaction (PCR) was positive with $<2000$ copies of deoxyribonucleic acid (DNA). Cerebrospinal fluid analysis was done, and it was positive for CMV IgG antibody and increased amount of proteins. He was treated with ganciclovir for acute CMV viremia and received three doses of intravenous immunoglobulin without much response. Patient had diffuse decrease in sensory and motor amplitudes, low normal conduction velocities, prolonged distal motor latencies in the left upper limb, and absent or prolonged F-wave responses on nerve conduction studies with severely decreased recruitment throughout, and a few positive sharp waves and fibrillation potentials on needle electromyography. These findings were most consistent with an acute diffuse neuropathy, most likely acute inflammatory demyelinating polyradiculopathy in early stage.

Day 4: Patient developed acute respiratory failure and was placed on mechanical ventilator.

Day 12: He demonstrated a slow and gradual improvement in his strength mostly in upper extremities after starting plasma exchange daily for 5 days.

Day 15: He was discharged to a rehabilitation facility. He had bifacial weakness; motor strength was $2 / 5$ in lower and $3 / 5$ in upper extremities. Sensations were intact.

Day 16: He developed acute onset of abdominal pain in left upper quadrant with high grade fever (103.4 F) and was admitted to the hospital. He was found to have dislodged gastrostomy tube, which was replaced by the interventional radiology. After that, the patient had an exploratory laparotomy. He was started on broad-spectrum antibiotics and culture was sent which grew coagulase negative staphylococcus. The patient continued to have high-grade fevers (102.9 F) despite exploratory laparotomy and aggressive wash out. Repeat CMV PCR was negative. Serum IgG ganglioside GQ1b antibody was $<1: 100$ titers.

Day 21: Repeat computed tomography (CT) abdomen showed modest sized splenic infarct [Figures 1 and 2] and prominent colitis. Patient had a small hypodense focus in the periphery of the spleen on the follow-up CT abdomen on day 25 and day 32, which was consistent with evolution of splenic infarct.

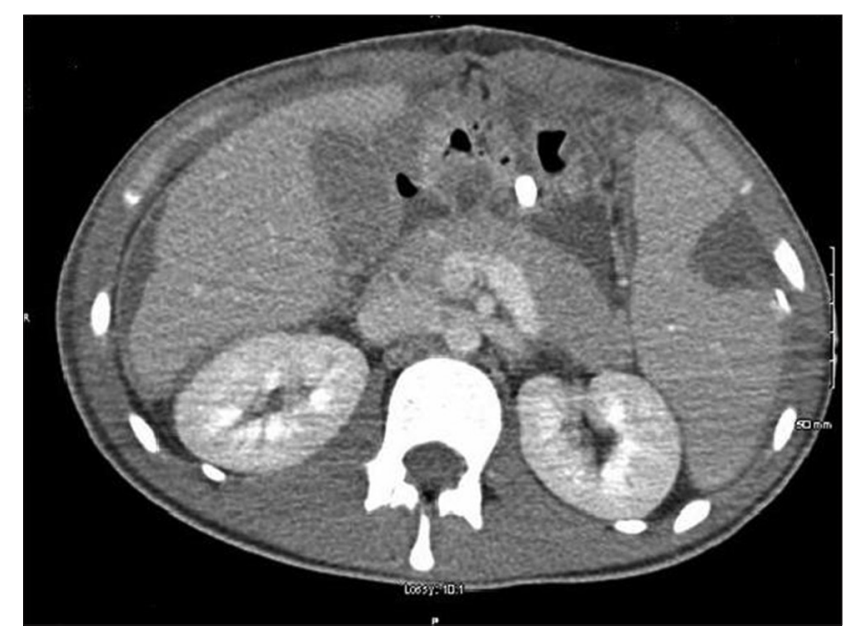

Figure 1: Horizontal section of CT abdomen showing splenic infarct

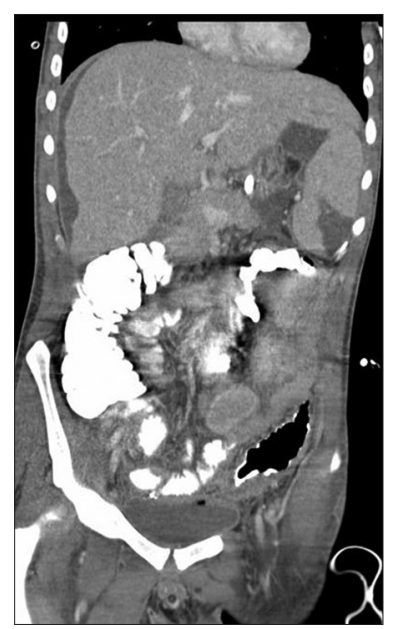

Figure 2: Coronal section of CT abdomen showing splenic infarct

Day 42: CT abdomen on the day of discharge showed decrease in the size of splenic infarct. Patient's condition was improved gradually and ultimately he was discharged to a rehabilitation facility.

\section{Discussion}

The clinical features of CMV infection in immunocompromised hosts have been extensively reported, but comparatively less attention has received for those observed in immunocompetent patients. Table 1 describes the clinical manifestations of CMV infection in immunocompetent patients. The diagnosis of acute CMV infection is based on positive CMV-specific IgG and IgM antibodies, peripheral blood positive for CMV pp65 antigens, and $\geq 600$ copies of CMV DNA/mL. ${ }^{[9]}$

Arterial or venous thrombosis in patients with acute CMV infection is not rare and is independent of the 
Table 1:Clinical manifestations of cytomegalovirus infection in immunocompetent patients

\begin{tabular}{|c|c|}
\hline Generalized symptoms & Fever, rash, sore throat ${ }^{[3-5]}$ \\
\hline Hematological & $\begin{array}{l}\text { Pancytopenia, lymphocytosis } \\
\text { (atypical lymphocytes), } \\
\text { monocytosis }{ }^{[3-5]}\end{array}$ \\
\hline Gastrointestinal system & Hepatitis, ${ }^{[6,7]}$ rarely colitis ${ }^{[3-5]}$ \\
\hline \multirow[t]{6}{*}{ Central nervous system ${ }^{[8]}$} & Retinitis \\
\hline & Meningitis \\
\hline & Encephalitis \\
\hline & Myelitis \\
\hline & Cranial nerve palsies \\
\hline & Guillain-Barre syndrome \\
\hline Respiratory system & Pneumonia $^{[3-5]}$ \\
\hline Arterial thrombosis ${ }^{[9]}$ & Splenic infarct, renal Infarct \\
\hline \multirow[t]{3}{*}{ Venous thrombosis ${ }^{[9]}$} & Deep vein thrombosis \\
\hline & Pulmonary embolism \\
\hline & Jugular vein thrombosis \\
\hline
\end{tabular}

typical risk factors for thrombosis. In a retrospective study by Atzmony et al. ${ }^{[9]}$ the incidence of thrombosis was $6.4 \%(n=9)$ in 140 patients with acute CMV infection. Splenic infarcts $(n=4)$ and renal infarct $(n=1)$ were the manifestations of arterial thrombosis. Venous thrombosis was presented as pulmonary embolism $(n=1)$, lower limb deep vein thrombosis $(n=1)$, upper limb deep vein thrombosis $(n=1)$, and jugular vein thrombosis $(n=1)$.

GBS is an acute demyelinating polyneuropathy that can occur after an infectious disease accounting for $60-70 \%$ of cases with CMV infection. ${ }^{[10]}$ Neurological involvement by CMV is usually attributed to aberrant immunological responses triggered by CMV. ${ }^{[1]}$ The outcomes have significantly improved in GBS patients with management in intensive care unit on ventilator support, the use of intravenous immunoglobulin, and plasmapheresis ${ }^{[12]}$ The diagnosis of CMV associated GBS was based on the presence of clinical features of acute onset of generalized weakness and numbness, which progress rapidly, together with facial diplegia and bulbar palsy; the cytoalbumino dissociation on cerebrospinal fluid analysis; the demyelinating motor and sensory neuropathy on electromyography; and serological evidence of increased IgM and IgG antibodies against CMV. Our patient was unique in that he developed splenic infarct and colitis after the initial episode of GBS and hepatitis.

In the prospective cohort study by Orlikowski et al., ${ }^{[2]}$ sensory deficits were observed in $72 \%$ of cases and facial palsy in $49 \%$ of cases, and test results positive for CMV DNA in $62 \%$ of cases tend to be associated with objective sensory defect $(P=0.052)$. The main factors associated with long-term neurological deficits (21\%) were older age $(P=0.001)$ and assisted ventilation during hospitalization $(P=0.005)$. Our patient had sensory deficits, positive for CMV DNA, and required the mechanical ventilation.

Two case reports highlighted the acute CMV patients presenting with bilateral abducens palsy ${ }^{[13,14]}$ In another case report patient had GBS along with periodontitis which may serve as a reservoir for CMV and its replication. ${ }^{[15]}$ Sinardi et al., ${ }^{[16]}$ reported an uncommon case of isolated vasculitis, restricted to the left sylvian artery in a patient with an autoimmune CMV associated GBS.

\section{Conclusion}

Multisystem complications can occur after CMV infection even in immunocompetent patients. Early recognition of complications is important and prompt treatment is critical to improve overall outcome. We cannot rule out a rare hereditary immunodeficiency in our patient. However, it is unlikely as the patient was healthy in the past.

\section{References}

1. Visser LH, van der Meche FG, Meulstee J, Rothbarth PP, Jacobs BC, Schmitz PI, et al. Cytomegalovirus infection and Guillain-Barre syndrome: The clinical, electrophysiologic, and prognostic features. Dutch Guillain-Barre Study Group. Neurology 1996;47:668-73.

2. Orlikowski D, Porcher R, Sivadon-Tardy V, Quincampoix JC, Raphael JC, Durand $\mathrm{MC}$, et al. Guillain-Barre syndrome following primary cytomegalovirus infection: A prospective cohort study. Clin Infect Dis 2011;52:837-44.

3. Vancikova Z, Dvorak P. Cytomegalovirus infection in immunocompetent and immunocompromised individuals: A review. Curr Drug Targets Immune Endocr Metabol Disord 2001;1:179-87.

4. Rafailidis PI, Mourtzoukou EG, Varbobitis IC, Falagas ME. Severe cytomegalovirus infection in apparently immunocompetent patients: A systematic review. Virol J 2008;5:47.

5. Taylor GH. Cytomegalovirus. Am Fam Physician 2003;67:519-24.

6. Azad AK, Ahmed T, Chowdhury AJ, Rahim MA, Mahmud AK, Rahman MA. Cytomegalovirus induced hepatitis in an immunocompetent host. Mymensingh Med J 2008;17:S104-6.

7. Mamun-Al-Mahtab, Rahman S, Khan M. Acute cytomegalovirus hepatitis in immunocompetent host. Kathmandu Univ Med J (KUMJ) 2009;7:79-81.

8. Eddleston M, Peacock S, Juniper M, Warrell DA. Severe cytomegalovirus infection in immunocompetent patients. Clin Infect Dis 1997;24:52-6.

9. Atzmony L, Halutz O, Avidor B, Finn T, Zimmerman O, Steinvil A, et al. Incidence of Cytomegalovirus-associated thrombosis and its risk factors: A case-control study. Thromb Res 2010;126:e439-43.

10. Kuwabara S. Guillain-Barre syndrome: Epidemiology, pathophysiology and management. Drugs 2004;64:597-610.

11. Hughes RA, Cornblath DR. Guillain-Barre syndrome. Lancet 2005;366:1653-66.

12. van der Meche FG, Schmitz PI. A randomized trial comparing intravenous immune globulin and plasma exchange in Guillain-Barre syndrome. Dutch Guillain-Barre Study Group. N Engl J Med 1992;326:1123-9.

13. Harada T, Kohriyama T, Ishizaki F, Nakamori K, Nakagawa Y, Ohtani $\mathrm{M}$, et al. Guillain-Barre syndrome and disturbance in multiple 
organs associated with cytomegalovirus infection. No To Shinkei 1990;42:245-51.

14. Kishi M, Sakakibara R, Ogawa E, Tateno F, Takahashi O, Koga M. Bilateral abducens palsy in a case of cytomegalovirus-associated Guillain-Barre syndrome. Neurol Sci 2011;32:1219-22.

15. Tabanella G, Nowzari H. Cytomegalovirus-associated periodontitis and guillain-barre syndrome. J Periodontol 2005;76:2306-11.
16. Sinardi D, Spada A, Marino A, Mondello E. A case of central nervous system vasculitis related to an episode of Guillain-Barrè syndrome. Crit Care 2000;4:245-7.

How to cite this article: Pulivarthi S, Gurram MK. A young patient with multisystem complications after cytomegalovirus infection. J Neurosci Rural Pract 2014;5:59-62.

Source of Support: Nil. Conflict of Interest: None declared.

\section{Author Help: Reference checking facility}

The manuscript system (www.journalonweb.com) allows the authors to check and verify the accuracy and style of references. The tool checks the references with PubMed as per a predefined style. Authors are encouraged to use this facility, before submitting articles to the journal.

- The style as well as bibliographic elements should be $100 \%$ accurate, to help get the references verified from the system. Even a single spelling error or addition of issue number/month of publication will lead to an error when verifying the reference.

- Example of a correct style

Sheahan P, O'leary G, Lee G, Fitzgibbon J. Cystic cervical metastases: Incidence and diagnosis using fine needle aspiration biopsy. Otolaryngol Head Neck Surg 2002;127:294-8.

- Only the references from journals indexed in PubMed will be checked.

- Enter each reference in new line, without a serial number.

- Add up to a maximum of 15 references at a time.

- If the reference is correct for its bibliographic elements and punctuations, it will be shown as CORRECT and a link to the correct article in PubMed will be given.

- If any of the bibliographic elements are missing, incorrect or extra (such as issue number), it will be shown as INCORRECT and link to possible articles in PubMed will be given. 